\title{
Hypergonadotropic Hypogonadism
}

National Cancer Institute

\section{Source}

National Cancer Institute. Hypergonadotropic Hypogonadism. NCI Thesaurus. Code

C113348.

Ovarian or testicular dysfunction associated with high levels of gonadotropins. 